\title{
An Open Source Contact-Free Palm Vein Recognition System
}

\author{
Ranjith Kumar M, Deepika G, Meenakshi Krishnan, Karthikeyan B \\ Embedded Systems Division, VIT University, Vellore, India
}

\begin{tabular}{l} 
Article Info \\
\hline Article history: \\
Received Sep 17, 2017 \\
Revised Nov 15, 2017 \\
Accepted Nov 22, 2017 \\
\hline Keyword: \\
Bag of visual words (BoVW) \\
K-means \\
Palm vein recognition \\
Scale invariant feature \\
Support vector machine (SVM) \\
Transform (SIFT) \\
\hline
\end{tabular}

\begin{abstract}
In this document, we propose a novel palm vein recognition system using open source hardware and software. We have developed an alternative preprocessing and feature extraction technique. The proposed system is built on Raspberry Pi using OpenCV 2.12. The palm vein image is cropped to Region of Interest(ROI) to reduce the computational time in real time systems and then preprocessed to enhance the vein pattern visibility and to extract more number of key points using SIFT algorithm. Then the descriptors are stored in a dictionary like codebook file during training. Later the descriptors are tested with unknown patterns. The clustering is based on $\mathrm{K}$-means algorithm and classification is done using Support Vector Machines $(\mathrm{SVM})$.
\end{abstract}

Copyright $@ 2017$ Institute of Advanced Engineering and Science. All rights reserved.

\section{Corresponding Author:}

Ranjith Kumar M,

Embedded Systems Division,

Vellore Institute of Technology (VIT) University, Vellore, India.

Email: ranjithkumar.m2015@vit.ac.in

\section{INTRODUCTION}

Ever since People wanted their belongings to be secure, modern advancements in science and technology have grown up bit by bit in the field of Biometrics. And the history of biometrics starts with the characteristics of fingerprint and later followed by other characteristics such as handwriting, face, retina, iris, voice and vein. All these human characteristics are patterns by nature and in most cases appear to be unique. Most of the biometric systems follow the conventional steps of processing the patterns. In this paper, we have come across the techniques used in finger vein, hand vein and palm vein (dorsal and ventral) patterns. The algorithm is implemented in a way so that the system behaves like a stand-alone real-time application. The entire system is built using low cost open source discrete elements. This system is built using Raspberry Pi, web camera, IR LEDs and OpenCV. The reason for choosing easily available components is that many people can contribute for the improvement of this technology and to provide new ways of improving the security of the system.

\section{CHARACTERISTICS OF VEIN PATTERNS}

Palm Vein provides high accuracy, high safety, high acceptance and permanence. It is immune to dirt, dust, dryness and moisture. This technique is hygienic and has less wear and tear because of its contactless nature. Palm vein patterns are complex as it has more than 5 million reference points. Palm vein is unique even among identical twins. Palm contains thicker veins than fingers and is easier to identify. Palm veins are insensitive against an environment (i.e., cold, temperature, creamy hands, skin scratches) and detectable only when blood is flowing.

When radiating a person's palm with near-infrared rays, palm vein pattern image can be captured. As veins are internal in the body and have unique differentiating characteristics, attempts to forge an identity are extremely difficult, thereby enabling a high level of security. There are two models of obtaining the vein 
pattern: reflection and transmission. In the reflection model, the camera and the IR source are placed facing the same direction. In the transmission model, the camera and the IR source are placed facing the opposite direction. The decision of choosing dorsal and ventral vein patterns depend on the amount of illumination required. Usually dorsal vein patterns appear easily but the reference points are comparatively low on the dorsum. So, it is preferable to choose ventral side of palm.

\section{SYSTEM DESIGN AND SOFTWARE SETUP}

Raspberry $\mathrm{Pi}$ is a pocket sized computer that can be customized for rapid prototyping. It comes in three different models with either Raspbian Wheezy or Raspbian Jessie. Raspbian OS was designed with high flexibility keeping electronic enthusiasts in mind. Tools like fswebcam, guvcview, gstreamer provide USB camera driver support for most of the devices.

The palm of a candidate should be illuminated using Infrared light. This source of IR spectrum can be produced using Gallium Arsenide (GaAs) Infrared LEDs of $850 \mathrm{~nm}$ or by placing a photographic (visible light) filter over the torch light opening. Several Researchers who have previously worked on the analysis of skin penetration depth have documented papers mentioning that $700 \mathrm{~nm}-900 \mathrm{~nm}$ is the range where the skin penetration is high. But practically speaking, any near Infrared LED will have an acceptable skin penetration depth. We have used Quantum QHM495LM Webcam and replaced the existing filter with a visible light filter. The QHM 495 LM comes with CMOS sensor of 25 Megapixels resolution and a refresh rate of $60 \mathrm{~Hz}$ with manual focus. The reason for choosing this camera is that CMOS sensors are better under low light conditions than CCD sensors. The QHM 495 LM has USB2.0 interface that can be connected to the Raspberry pi.

\section{IMAGE ACQUISITION}

The vein pattern of a human cannot be captured using an ordinary camera because the patterns are not visible in the visible region. Therefore, we use an Infrared camera to capture the vein pattern. This Infrared camera has to operate in the near infrared region of the frequency spectrum [1]-[3]. The frequency in which it operates has to be chosen based on the skin penetration depth. From the previous works carried out by various researchers, it has been observed that the image has to be captured in the range of $800 \mathrm{~nm}$ to 900 $\mathrm{nm}$ for better details of the image. As the Near-Infrared Cameras are expensive, we modify an ordinary camera into a Near-Infrared Camera. This is done by replacing the existing Infrared filter in an ordinary camera by a visible light filter. This blocks the visible light spectrum and allows the camera to capture the image in Near-Infrared spectrum [17]-[18]. For testing purposes, we have used CASIA Palm print database as shown in Figure 1.

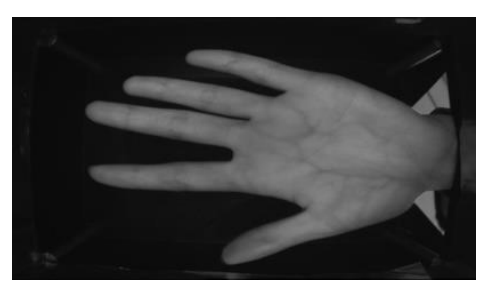

Figure 1. Acquired Image

The acquired image has to be processed so that the matching has to be invariant to parameters such as rotation, scale, brightness and contrast. For this purpose, we will segment the Region of Interest (ROI) [4]-[6] of the image. The ROI can be cropped from the acquired image by several ways. In an automated system, the algorithm has to be adaptive so that any sample of the same candidate should have the same ROI every time. In the proposed method, the algorithm works as follows:

Proposed_Algorithm_ROI:

a. Find the contours (Object Boundary) in the acquired image.

b. Find the convex hull for the hand contour.

c. Find the convex defects (Nothing but the interest points to crop the ROI) of the image from the palm contour and the convex hull whose depth is greater than the threshold. 
d. Crop the image (ROI) with respect to the interest points.

The ROI region with respect to the interest points is shown in Figure 2. The resolution of the ROI can be chosen in an optimal way, so that the system can have tradeoff between computation (processing) time and Receiver Operating Characteristic (ROC) curve. ${ }^{[7][8]}$ In some of the previous researches, the ROI is not extracted from the acquired image. There are various pros and cons of doing so. The comparison of the two ways of processing i.e. processing without ROI segmentation and processing the extracted ROI is tabulated below. The segmented ROI is shown in Figure 3. In our implementation, the ROI size is chosen to be $160 * 160$.

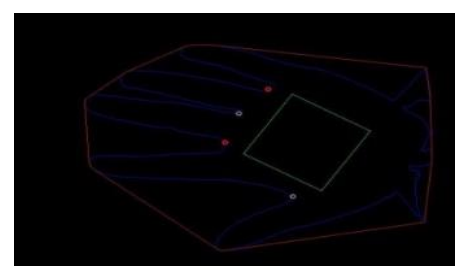

Figure 2. Region of Interest with Respect to the Interest Points

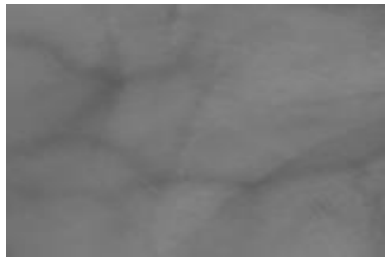

Figure 3. Segmented ROI

\begin{tabular}{|c|c|c|}
\hline S1. No. & Processing without ROI segmentation & Processing the extracted ROI \\
\hline 1. & Susceptible to rotation, scaling, etc., & Scaling and rotation tolerant \\
\hline 3. & $\begin{array}{l}\text { Presence of environmental disturbances in the } \\
\text { background could cause an undesired behavior in the } \\
\text { system }\end{array}$ & $\begin{array}{l}\text { System is stable because it avoids the environmental } \\
\text { disturbances in the background }\end{array}$ \\
\hline 4. & More number of feature points & Less number of feature points \\
\hline 5. & $\begin{array}{l}\text { Computation over headmay exist in real time } \\
\text { environment }\end{array}$ & Computation over head is reduced \\
\hline
\end{tabular}

\section{IMAGE PRE-PROCESSING}

The ROI image is pre-processed to obtain a clear pattern for the feature extraction.

\subsection{Histogram Equalization}

Histogram equalization is technique wherein the contrast of the is enhanced by altering the intensity levels of the pixels. The histogram equalization formula for ROI image is

$$
\text { HE(ROI) }=\operatorname{Round}\left(\frac{(\text { CDF(ROI) }- \text { CDFmin })}{(\text { ImageSize })-\text { CDFmin }} \times(L-1)\right)
$$

Where $\mathrm{L}$ is the intensity value of the image and CDFmin is the minimum non-zero value of cumulative distribution function which is also an image accumulated normalized histogram. Therefore, applying histogram equalization on the ROI image will lower the contrast of the vein patterns and increase the contrast of the background. The histogram equalized image is shown in Figure 4.

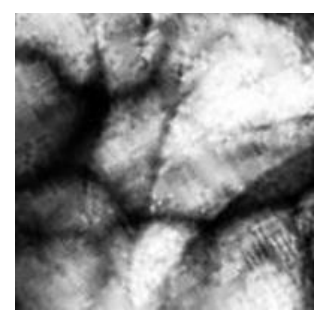

Figure 4. Histogram equalized image

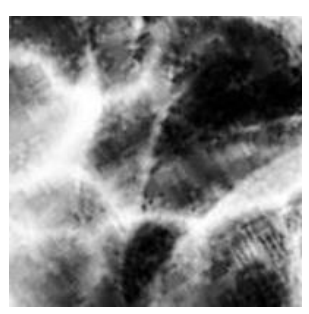

Figure 5. Image negative 


\subsection{Image Negative}

Negative of an image is nothing but making all dark areas to appear light and light areas appear dark. It is basically inversion of an image. This inversion of a gray scale image can be easily accomplished by subtracting each pixel intensity value from the highest intensity value in the image. Thus by subtracting each pixel intensity value from 255 , we can generate the negative of an image.

Image Negative $=(\mathrm{L}-1)-\mathrm{HE}$

Where $\mathrm{L}$ is the intensity value of the image. Taking negative of the histogram equalized image will therefore make the low contrast vein patterns appear are dark lines and the remaining region appears light. The Image negative output is shown in Figure 5.

\subsection{Erosion}

Erosion is a basic morphological operation used for removal of pixels from object boundaries. Thus erosion can be used to shrink the size of an object in an image and to remove the unnecessary details. We apply erosion process on the inverted ROI image to remove the unnecessary details and to further enhance the appearance of the vein patterns. The eroded image is shown in Figure 6.

\subsection{Skeletonization}

Skeletonization is a process used to reduce the thickness of the foreground region to appear as a thin line. We skeletonize the eroded image to obtain thin lines for vein patterns so that the feature extraction process becomes easier. The skeletonized image is shown in Figure 7.

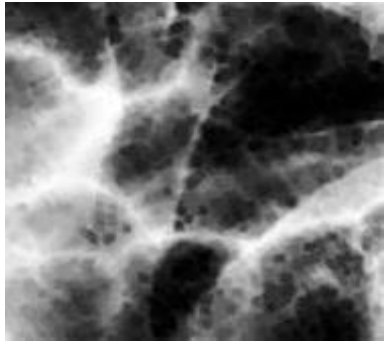

Figure 6. Eroded Image

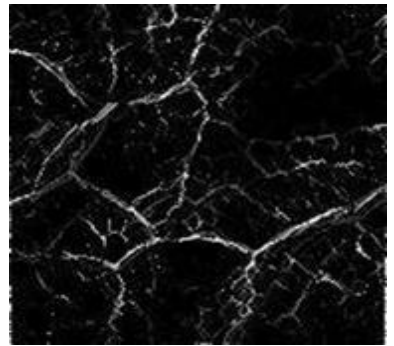

Figure 7. Skeletonized Image

\section{FEATURE EXTRACTION \\ 6.1. SIFT Feature Descriptor}

The SIFT feature descriptor algorithm is applied to the preprocessed ROI image to find the robust feature points. There are different variants of SIFT proposed by various researchers [9]-[11]. It is a robust method than ORB and SURF feature descriptor and mainly used for object recognition. We have implemented Robert Lowe's implementation of SIFT. The SIFT key points are shown in Figure 8.

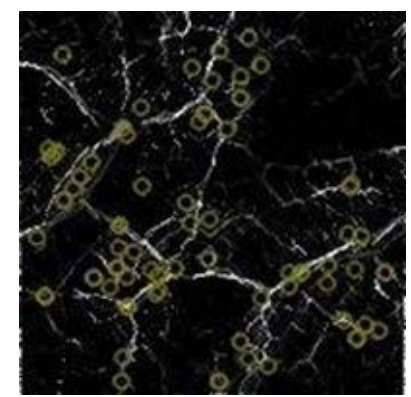

Figure 8. Key points extracted from SIFT 


\section{MATCHING}

\subsection{Bag of Features}

Bag of features model also called as Bag of visual words model is basically used for image classification where the image features are considered as words. To represent an image using the BoF model, 3 steps are to be followed- feature extraction, feature description and codebook generation. BoVW model uses K-means clustering to generate the codebook [12]-[13].

\subsection{Support Vector Machines (SVM)}

SVM is a statistical property based supervised learning algorithm used for image classification by plotting points on a hyper plane. ${ }^{[14][15][16]}$ It performs complex data transformations based on something called as the kernel trick. It then determines an optimal boundary based on these transformed data

\section{RESULTS}

The images were trained using random datasets and tested with unknown inputs. There were 100 classes to be classified. The class indices start from 0 to 99 i.e If a person of $9^{\text {th }}$ class is recognized, the algorithm will return " 8 " which denotes the class number. The result is shown in Figure 9.

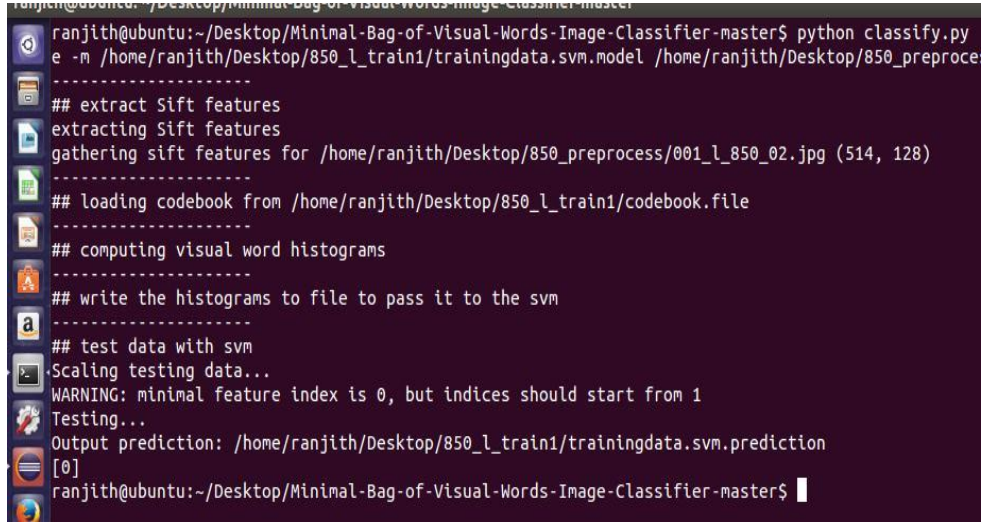

Figure 9. Recognition Result

\section{REFERENCES}

[1] AliMohsin Al-juboori, Wei Bu, Xiangqian Wu and Qiushi Zha, "Palm Vein Verification Using Multiple Features and Locality Preserving Projections," The Scientific World Journal, Article ID 246083, Volume 2014.

[2] Sakthivel G, "Hand Vein Detection using Infrared Light for Web based Account," International Journal of Computer Applications (0975 - 8887) Volume 112 - No 10, February 2015

[3] Wenxiong Kang, "Vein pattern extraction based on vectorgrams of maximal intra-neighbor difference," Pattern Recognition Letters 33 (2012) 1916-1923 Elsevier, 10 March 2012.

[4] Omidiora Elijah Olusayo, Oladosu John Babalola, Ismaila Wasiu Oladimeji, "Palm Vein Recognition System Using Hybrid Principal Component Analysis and Artificial Neural Network," International Journal of Advanced Research in Computer Science and Software Engineering, Volume 3, Issue 7, July 2013.

[5] Wenxiong Kang1, Yang Liu, Qiuxia Wu, Xishun Yue, "Contact-Free Palm-Vein Recognition Based on Local Invariant Features," PLOS ONE 9(5): e97548. doi:10.1371/journal, May 27, 2014.

[6] Faritha Nasreen, Arul Dhana Saam Prakash, Aparrnaa Raghuraman, "Versatile and Economical Acquisition Setup for Dorsa Palm Vein Authentication," Published by Elsevier B.V, 2015.

[7] Zahra Honarpisheh, Karim Faez, "An Efficient Dorsal Hand Vein Recognition Based on Firefly Algorithm," International Journal of Electrical and Computer Engineering (IJECE) Vol 3, No 1: February 2013, pp. 30 41.

[8] Yingbo Zhou, Ajay Kumar, "Contactless Palm Vein Identification using Multiple Representations", IEEE 2010.

[9] Deepak Prasanna.R, Neelamegam.P, Sriram.S, Nagarajan Raju, "Enhancement of vein patterns in hand image for biometric and biomedical application using various image enhancement techniques," International conference on modeling optimization and computing, 2012.

[10] Jason Forté, "Development of a Near Infrared Hand Vein Imaging Device with Software Enhancement," November 2014

[11] Jen-Chun Lee, "A novel biometric system based on palm vein image," Pattern Recognition Letters 33 (2012) 15201528, Elsevier, 25 April 2012. 
[12] Mona A. Ahmed, Hala M. Ebied, El-Sayed M. El-Horbaty, AbdelBadeeh M. Salem, "Analysis of Palm Vein Pattern Recognition Algorithms and Systems," International Journal of Bio-Medical Informatics and e-Health, Volume 1, No.1, June - July 2013.

[13] Centre du Parc, Rue Marconi 19, CH-1920 Martigny, "Palm Vein Database and Experimental Framework for Reproducible Research,” 2015.

[14] Anika Pflug, Daniel Hartung, Christoph Busch, "Feature extraction from vein images using spatial information and chain codes," Elsevier, 2012.

[15] Mr. Vishal U. Bhosale, Mr. Onkar S. Kale, Mr. Mahesh W. Pawar, Mr. Roshan R. Patil, Mr. Pritam S. Patil, Prof Mrs. Sonali Madankar, "Palm Vein Extraction and Matching for Personal Identification," IOSR Journal of Computer Engineering (IOSR-JCE) e-ISSN: 2278-0661, pISSN: 2278-8727Volume 16, Issue 2, Ver. IX (Mar-Apr. 2014).

[16] Gitanjali Sikka, Er. Vikas Wasson, "Palm Vein AuthenticationReview," International Journal of Science and Research (IJSR), Volume 3 Issue 9, September 2014.

[17] Kuang-Shyr Wua, Jen-Chun Lee, Tsung-Ming Lo, Ko-Chin Chang, Chien-Ping Changa, "A secure palm vein recognition system," The Journal of Systems and Software 86 (2013) 2870- 2876, Elsevier, 2013.

[18] Gayathri S, K Gerard Joe Nigel,S Prabakar, "Low Cost Hand Vein Authentication System on Embedded Linux Platform," International Journal of Innovative Technology and Exploring Engineering (IJITEE), ISSN: 2278-3075, Volume-2, Issue-4, March 2013. 